\title{
Laboratory Results from Dielectric Spectroscopy of Field Aged XLPE Cables with Respect to Water Trees
}

Pedersen, Kenneth; Sedding, Howard; Fenger, Mark; Henriksen, Mogens; Holbøll, Joachim

Published in:

IEEE International Symposium on Electrical Insulation 2006

Link to article, DOI:

10.1109/ELINSL.2006.1665368

Publication date:

2006

Document Version

Publisher's PDF, also known as Version of record

Link back to DTU Orbit

Citation (APA):

Pedersen, K., Sedding, H., Fenger, M., Henriksen, M., \& Holbøll, J. (2006). Laboratory Results from Dielectric Spectroscopy of Field Aged XLPE Cables with Respect to Water Trees. In IEEE International Symposium on Electrical Insulation 2006 (pp. 509-514). IEEE. https://doi.org/10.1109/ELINSL.2006.1665368

\section{General rights}

Copyright and moral rights for the publications made accessible in the public portal are retained by the authors and/or other copyright owners and it is a condition of accessing publications that users recognise and abide by the legal requirements associated with these rights.

- Users may download and print one copy of any publication from the public portal for the purpose of private study or research.

- You may not further distribute the material or use it for any profit-making activity or commercial gain

- You may freely distribute the URL identifying the publication in the public portal 


\section{Laboratory Results from Dielectric Spectroscopy of Field Aged XLPE Cables with Respect to Water Trees}

\author{
Kenneth Pedersen \\ Ørsted $\cdot$ DTU \\ Technical University of Denmark \\ Building 325, 2800 Lyngby, Denmark \\ kp@oersted.dtu.dk
}

\author{
Howard Sedding, Mark Fenger \\ Kinectrics Inc., 800 Kipling Avenue \\ Toronto, Ontario M8Z 6C4, CANADA \\ Howard.sedding@kinectrics.com \\ mark.fenger@kinectrics.com
}

\author{
Joachim Holboell, Mogens Henriksen \\ Ørsted·DTU \\ Technical University of Denmark \\ Building 325, 2800 Lyngby, Denmark \\ jh@oersted.dtu.dk,mh@oersted.dtu.dk
}

\begin{abstract}
This paper deals with the application of frequency domain dielectric spectroscopy for the detection of water trees in XLPE cables. During the research, dielectric spectroscopy measurements have been carried out on cable samples with and without water trees while it was attempted to preserve many of the uncertainties present in the field. The experiments support the applicability of a high voltage test developed primarily at KTH in Sweden, which utilizes the nonlinearity of the dissipation factor. Subsequently it was investigated whether this high voltage method also seems reliable at low voltages. It turned out that the fundamentals of the high voltage method were not directly applicable at low voltages as the nonlinearity of the permittivity is not noticeable at low voltages, at least compared to the deviations. Instead a novel method for interpreting the low frequency measurements is proposed though it needs further verification. Finally a theoretically possible modification of the measurement system is proposed, which deals with the problem of a limited current capability of the amplifier.
\end{abstract}

\section{INTRODUCTION}

Water trees are the main reason for breakdown in older XLPE cables, and though the relation between water trees and breakdown strength of XLPE cables is not fully understood, good correlations has been found between the breakdown strength and parameters such as dielectric losses expressed by $\varepsilon "$ (and thus $\tan \delta$ ) [1-4] and the length of the longest water tree [3]. The water tight design was first introduced around 1990 so there are still a lot of cables in service, which may be severely water treed.

On the one hand it is preferable to replace cables before breakdown occur, but at the same time it may be uneconomical to replace cables much too early. Therefore, reliable and accurate condition assessments of XLPE cables with respect to water tree detection are important.

Though some publications exist on utilization of the nonlinearity of frequency domain dielectric spectroscopy with respect to water tree detection in XLPE cables [1-7], it is still regarded as a fairly new method according to a recent survey [8] and thus further verification should be appreciated.

A recent conference paper [6] divide cables into $1^{\text {st }}, 2^{\text {nd }}$ and $3^{\text {rd }}$ generation XLPE cables and discusses experimental results from field aged $2^{\text {nd }}$ generation cables. $1^{\text {st }}$ generation refers to those cables which were produced without an extruded insulation screen. In the 1970's the extruded insulation screen was introduced, which characterizes the $2^{\text {nd }}$ generation. The $3^{\text {rd }}$ generation refers to the water-tight design introduced in the 1990 's. The conclusion was that $2^{\text {nd }}$ generation cables showed only small signs of nonlinearity, corresponding to a voltage dependent permittivity (VDP) and thus the 'usual' method described thoroughly in [4] doesn't seem reliable for this type of cable unless water trees are completely spanning the insulation. In this case a leakage current response was obtained. The less significant VDP response is due to a much smaller water tree density in $2^{\text {nd }}$ generation cables. This actualizes the need of additional experiments on field aged cables as for some cable types it may be difficult to distinguish between $1^{\text {st }}$ and $2^{\text {nd }}$ generation cables and this may be complicated further by geographical differences as the measurements related to the work at $\mathrm{KTH}$ were carried out mainly on cables in Scandinavia.

This paper addresses the need for such additional verification with respect to medium voltage cables. Moreover it has been investigated whether the VDP approach can be carried out at lower voltages and still gives reliable results. This would be a great advantage since there are some practical issues with the high voltage method. First of all, for the high voltage method a high voltage amplifier is needed which makes the equipment much less compact and thus less flexible since the maximum amplifier output current limits the maximum length of the cable to be tested. High voltage measurements also increase the risk of human injuries. At the end of this paper a proposal is given to improve the current capability of the measurement setup.

\section{EXPERIMENTAL SETUP}

\section{A. Overview}

Measurements were carried out on six field aged North American cable samples, which had been dried previously. 15 and $25 \mathrm{kV}$ samples were tested, all single phase with a shield consisting of strands without a surrounding sheath.

Subsequently the cables were placed in a water tank and exposed to $8 \mathrm{kV}$ in order to speed up the water tree regeneration. Measurements were carried out several times during the ageing process. Besides these measurements, measurements were also carried out on a new cable for comparison. The cables applied in the present research are 
regarded as belonging to the $1^{\text {st }}$ generation and they have been in the ground for around 20-25 years.

For convenience the cables are called P1, P2, P3, W1, W2, $\mathrm{W} 3$ and N1. The latter cable, N1, is the new cable. P1-P3 are quite similar as the shield conductors are rather loose and thus it is likely that water trees can quickly regenerate. In contrast to these, the cables W1-W3 have strands which sit much closer together. Figure 1 and 2 shows $\mathrm{P} 3$ and $\mathrm{W} 1$ respectively. $\mathrm{P} 1$ and $\mathrm{P} 2$ are $15 \mathrm{kV}$ cables of the same design and manufacturer, whereas P3 is to some extend similar though it is a $25 \mathrm{kV}$ cable. $\mathrm{W} 1-\mathrm{W} 3$ are $25 \mathrm{kV}$ cables.

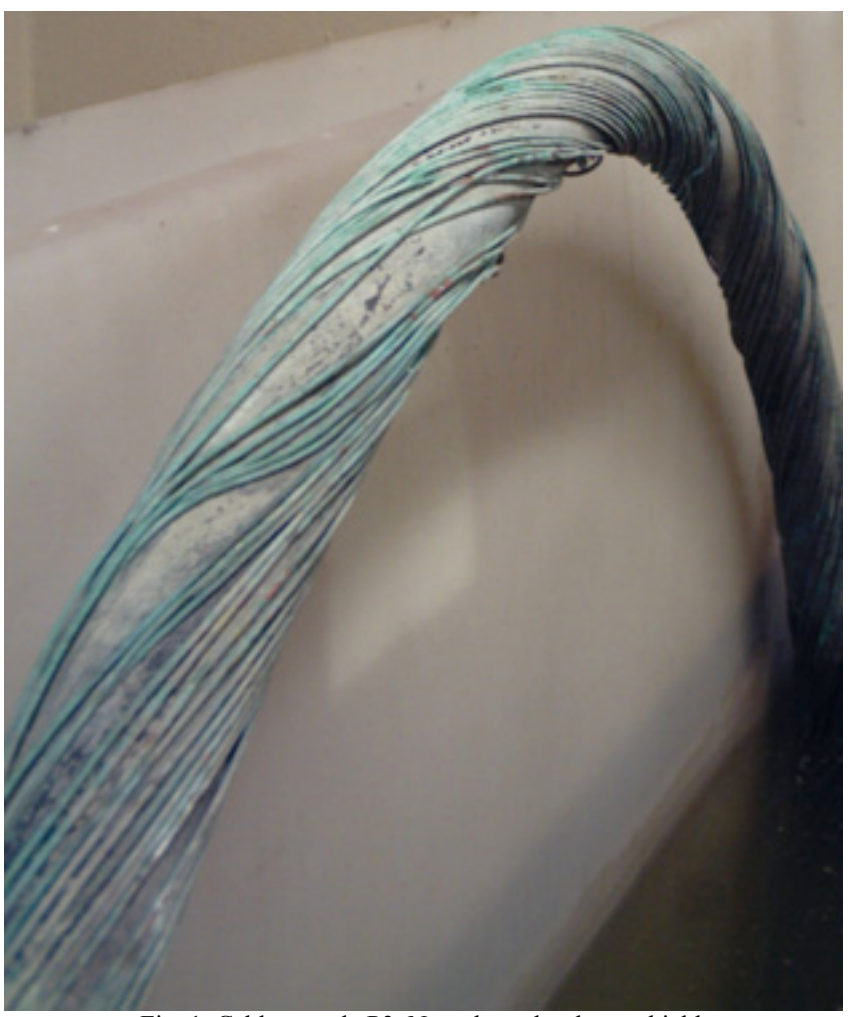

Fig. 1. Cable sample P3. Note the rather loose shield.

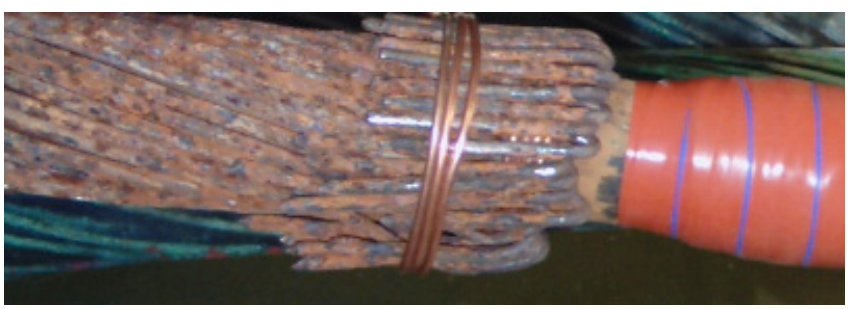

Fig. 2. Cable sample W1. The strands of the shield conductor are much closer together on this cable.

\section{B. Equipment}

The applied measurement system was the IDA 200 from Programma (now a subdivision of GE). This equipment specializes in frequency domain dielectric measurements at low frequencies.
For high voltage measurements a TREK 20/20A amplifier was used as high voltage supply. As the IDA 200 was designed for a specific high voltage unit called the IDA HVU, a special interface was also needed in order to connect the TREK to the IDA 200.

\section{High Voltage Method}

\section{A. Theory}

Before the measurements are presented, the VDP method is presented below with abbreviations adopted from [2, 4, 7]:

As mentioned in the introduction, water treed XLPE exhibits a voltage dependent permittivity VDP. If no water trees are present the losses will be lower and the permittivity is expected to be linear. This type of behavior is denoted as a "linear low loss permittivity" LLLP. Besides ranking cables from the degree of VDP, they might be ranked according to the voltage level where the frequency response of the permittivity changes characteristics to that of a leakage current. This type of response is denoted as a "transition to leakage current" response (TLC).

The leakage current response can be explained by considering an open-ended typical cable with a limited length, which means that the transmission line properties of the cable can be ignored at low frequencies and thus the cable can be modeled as a capacitance. Cable and model are illustrated in figure 3 .

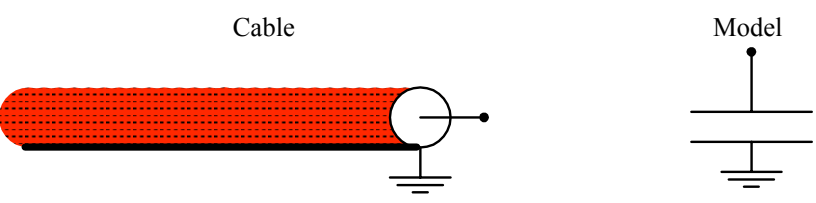

Fig. 3. Cable and corresponding capacitance model.

In case of very long cables or relatively high frequencies the transmission line properties can be accounted for by measuring the inductance and resistance of the cable by additional measurements. However, Werelius showed [4] that the lumped capacitor approach only gives a deviation in $\tan \delta$ of $10^{-5}$ at $50 \mathrm{~Hz}$ for a typical cable of 1000 meters, so for most purposes the transmission line properties can be ignored.

Furthermore, if we assume that the XLPE is not deteriorated, $\tan \delta$ will only have a weak frequency dependency [4,7]. The input admittance of the cable can thus be written as

$Y_{\text {in }}=j \cdot \omega \cdot C=j \cdot \omega \cdot C^{\prime}+\omega \cdot C^{\prime}, \quad C=C^{\prime}-j \cdot C^{\prime}$,

corresponding to the dissipation factor

$\tan \delta=\operatorname{Real}\left[Y_{i n}\right] / \operatorname{Imag}\left[Y_{i n}\right]=C{ }^{\prime}, / C^{\prime}=\varepsilon^{\prime}, / \varepsilon^{\prime}$.

Here $\varepsilon$ ' and $\varepsilon$ '" represents the real and imaginary part of the effective relative permittivity of the cable capacitance which, depending on the cable design, may or may not be a reasonable approximation to the relative permittivity of the XLPE, as the measured $\tan \delta$ is also influenced by other 
materials such as the semicon. In case it is a reasonable approximation, $\varepsilon$ ' can be computed from $\tan \delta$ as $\varepsilon$ ' is approximately 2.3 for XLPE, i.e.

$\varepsilon "=2.3 \cdot \tan \delta . \quad$ (assuming no frequency dependence)

In this paper, these assumptions are applied where ever statements regarding $\varepsilon$ ', of the cable samples are given.

If one or more leakage currents are present they can be modeled by a conductance in parallel with the capacitor. As admittances in parallel can be added, the input admittance becomes:

$Y_{\text {in }}=j \cdot \omega \cdot C=j \cdot \omega \cdot C^{\prime}+\omega \cdot C{ }^{\prime}+G_{\text {leakage }}$

corresponding to the dissipation factor

$\tan \delta=\operatorname{Real}\left[Y_{\text {in }}\right] / \operatorname{Imag}\left[Y_{\text {in }}\right]=\left(G_{\text {leakage }}+\omega \cdot C^{\prime \prime}\right) /\left(\omega \cdot C^{\prime}\right)$.

When the frequency becomes small the leakage current will dominate the loss part of the admittance and $\tan \delta$ converges:

$\tan \delta \approx G_{\text {leakage }} /\left(\omega \cdot C^{\prime}\right)$

for small values of $\omega$. Thus the following relation will hold:

$\tan \delta(\omega / 10) \approx 10 \cdot \tan \delta(\omega)$

for small values of $\omega$.

The relation (7) corresponds to an approximately straight line when plotting $\tan \delta(\mathrm{f})$ in a log-log plot, and the slope of this straight line equals $-1 \mathrm{~dB} / \mathrm{dB}$. This is illustrated in fig. 4 where a moist string was inserted between the conductor and ground during a measurement.

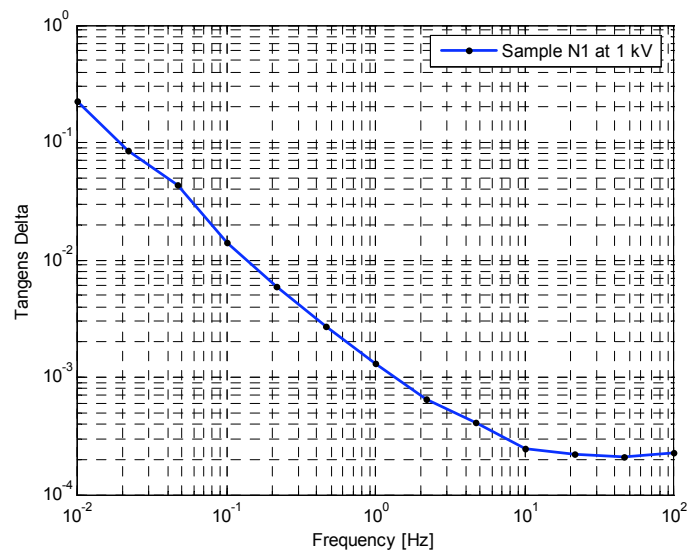

Fig. 4. $\tan \delta(f)$ for sample N1 with added leakage path.

If the response jumps from a rather flat response to that of a leakage current when the voltage is increased, it is said that the cable has a "transition to leakage current" response (TLC) [4]. If the leakage current response is obtained at the lowest voltage included in a measurement series, the response is simply called a "leakage current" response (LC).

In the Botkyrka project [7] the breakdown strengths of cables with LC and TLC responses were estimated to be below 2.5 times the service voltage. The breakdown strength of cables with VDP responses were estimated to be between 2.5 and 4 times the service voltage. For cables with an LLLP response the breakdown strength was estimated to be above 4 times the service voltage. Besides the above classification, the cables with VDP response were ranked with respect to the degree of nonlinearity in [7].

\section{B. Quantitative criteria for determining VDP}

Due to uncertainties in the measurements it is useful to apply quantitative criteria for separating a VDP response from a LLLP response. In [7] the following criteria were applied in order to clearly identify a VDP response:

$$
\begin{aligned}
& \varepsilon^{\prime}{ }^{\prime}>8 \cdot 10^{-4} \text { at service voltage } \\
& \Delta \varepsilon^{\prime}{ }^{\prime}{ }_{\text {nonlin }}>1 \cdot 10^{-4} \\
& \Delta \varepsilon^{\prime}{ }_{\text {nonlin }}>2 \cdot 10^{-4}
\end{aligned}
$$

Here $\Delta \varepsilon^{\prime}{ }_{\text {nonlin }}$ and $\Delta \varepsilon{ }^{\prime}{ }_{\text {nonlin }}$ are the increases in the real and the imaginary part respectively of the relative permittivity when increasing the voltage from half the service voltage to the service voltage. Converted to $\tan \delta$ values with the aid of (3), the two first criteria become:

$$
\begin{aligned}
& \tan \delta>3.5 \cdot 10^{-4} \text { at service voltage } \\
& \Delta_{\text {nonlin }}(\tan \delta)>4.3 \cdot 10^{-5}
\end{aligned}
$$

The criteria regarding $\varepsilon$ ', wasn't investigated in this study.

\section{High voltage results - visual evaluation}

In figures 5-7 the responses of P1-P3 are shown. Important details regarding the measurements are presented below and the results are discussed. Figures 5-7 contain the final responses of P1-P3 after approximately 400 hours of ageing at $8 \mathrm{kV}$. The ageing voltage of $8 \mathrm{kV}$ is close to the phase-toground service voltage of $\mathrm{P} 1$ and $\mathrm{P} 2$ which is $15 \mathrm{kV} / \sqrt{3}$. For each voltage the frequency was swept before proceeding to a higher voltage.

A brief visual evaluation concludes that the nonlinearity is not especially significant below the service voltage of P1 and $\mathrm{P} 2$ though it is quite stable for P1 and P2 in the sense that the relative increase in $\tan \delta$ due to an increase in voltage is always positive and approximately frequency independent, except at the lowest frequencies. This alone indicates slight water treeing though the quantitative criteria should be applied in order to determine for sure whether we are dealing with a VDP response. This is done in section D.

Furthermore: Above the service voltage P1 exhibits a very strong VDP response, which is approximately frequency independent. The independence of frequency means that leakage currents cannot explain this behavior. Thus P1 must be regarded as a significantly water treed cable. 


\section{High voltage results - Quantitative evaluation}

The first criteria to be fulfilled in order to determine whether we are dealing with VDP response is that $\tan \delta$ is above $3.5 \cdot 10^{-4}$. This criterion was only fulfilled for P1-P3 which is why the results of W1-W3 and N1 are not discussed further.

In this study it has been found that the hysteretic effects are significant, i.e. the permittivity increases with increasing voltage but when the voltage is decreased again, the losses stay up.

This can very well explain why the voltage dependency is not significant at voltages below the applied ageing voltage of $8 \mathrm{kV}$, which was applied shortly before the measurements were carried out, and thus why the quantitative criteria for $\Delta_{\text {nonlin }}(\tan \delta)$ is not fulfilled for all frequencies.

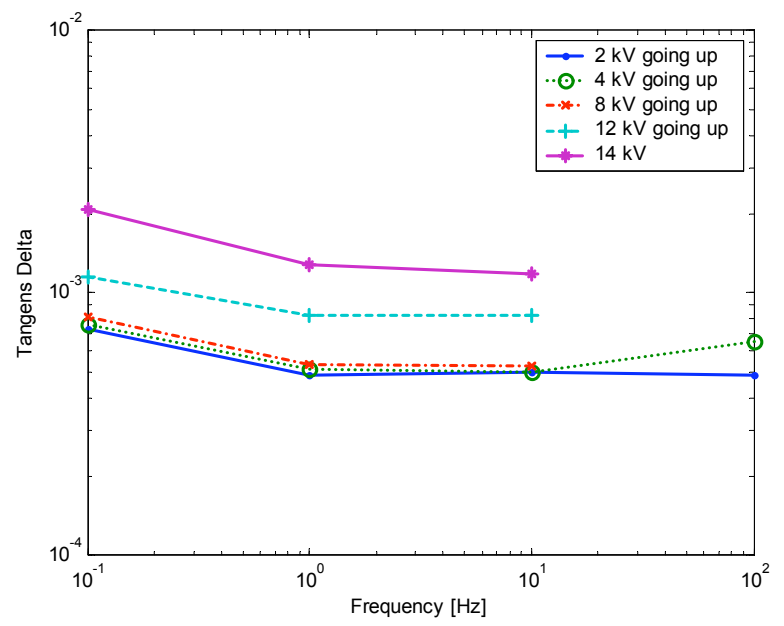

Fig. 5. Results from cable P1, a $15 \mathrm{kV}$ cable.

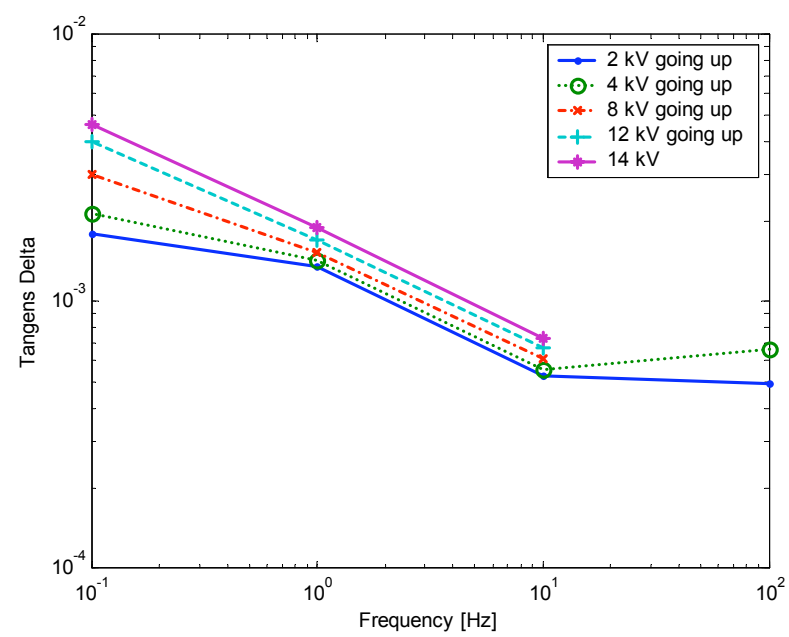

Fig. 6. Results from cable P2, a $15 \mathrm{kV}$ cable.
The criteria for $\Delta_{\text {nonlin }}(\tan \delta)$ is fulfilled for P1-P3 at $0.1 \mathrm{~Hz}$. However, as can be seen in the plots, the frequency responses are definitely not flat. Instead $\tan \delta$ as well as the degree of nonlinearity increases as the frequency decreases. This type of frequency dependent nonlinearity can be explained by voltage dependent leakage currents at the cable terminations. This explanation is supported by [4], which concludes that some terminations have a voltage dependent influence on the response and that the influence decays with frequency. For this reason, and the fact that the available samples are rather short, it was in this study chosen to measure a wider frequency band in order to distinguish the voltage dependence from end effects. In the field the cables will be longer so lower frequencies should be sufficient.

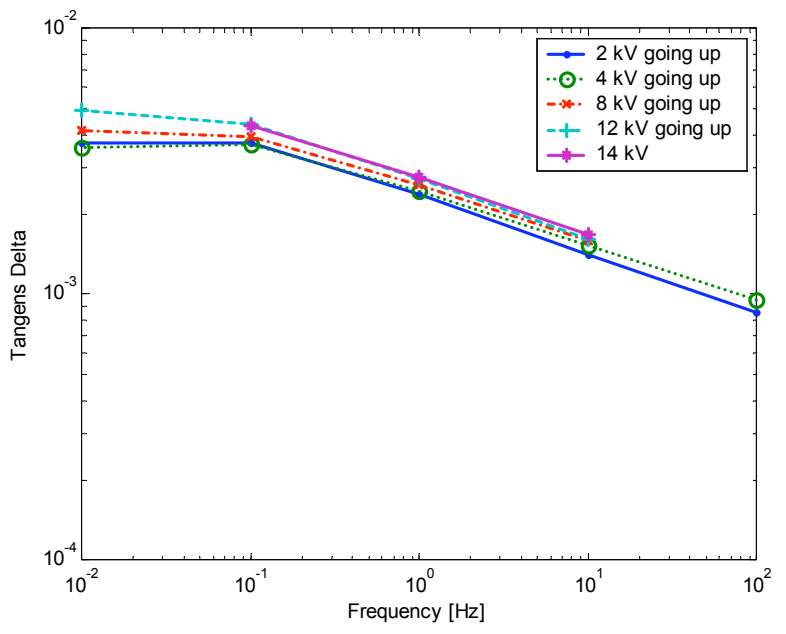

Fig. 7. Results from cable P3, a $25 \mathrm{kV}$ cable.

It was then investigated theoretically whether the observed voltage dependency at $10 \mathrm{~Hz}$ can be caused by a voltage dependent surface conductivity at the terminations, as guarding was not applied. This investigation was based on the following assumptions:

- The deviation between $\tan \delta$ at $14 \mathrm{kV}, 0.1 \mathrm{~Hz}$ and tan $\delta$ at $1 \mathrm{kV}, 10 \mathrm{~Hz}$ is solely caused by the voltage dependency of the conductivity at the terminals (worst case).

- The loss angle at $1 \mathrm{kV}, 10 \mathrm{~Hz}$ is identical to the loss angle of the XLPE, i.e. other losses are ignored.

- All parameters are assumed to be frequency independent.

With these assumptions the nonlinearity caused by the termination can be computed at $10 \mathrm{~Hz}$ where it was found to be hardly visible. Thus, the nonlinearities are likely to be caused by water trees and not nonlinear terminations. However, the measurements below service voltage at $10 \mathrm{~Hz}$ do not fulfill the quantitative criteria for $\Delta_{\text {nonlin }}(\tan \delta)$.

As mentioned earlier this may be due to the hysteretic effects as the criteria is fulfilled for both P1 and P2 when going from $8 \mathrm{kV}$ to $14 \mathrm{kV}$ at $10 \mathrm{~Hz}$. However, the criteria are useful for separating true nonlinearity from variations caused 
by other effects such as time dependence so that healthy cables are not diagnosed as slightly water treed. The presented results were quite reliable and thus the criteria may be harder than necessary for this study. Earlier measurements were less reliable but at the end of this study it was found that ageing voltage of approximately $2 \mathrm{kV}$ for a few hours increased the reliability of the measurements significant.

Above $8 \mathrm{kV}$ the nonlinearity of $\mathrm{P} 1$ is highly pronounced compared to $\mathrm{P} 2$. As $\mathrm{P} 1$ and $\mathrm{P} 2$ have identical designs, $\mathrm{P} 1$ is regarded as being significantly more water treed than P2. P3 may also be water treed but as $\mathrm{P} 3$ is a $25 \mathrm{kV}$ cable, the equipment was not able to go beyond the service voltage and thus, the internal fields must on average have been smaller for P3 than for P1 and P2 during the measurements. This may explain why the nonlinearity was not very significant for P3.

\section{Low Voltage Method}

Frequency domain dielectric spectroscopy at high voltages has two clear advantages compared to a corresponding low voltage method:

1. VDP responses stand out clearer.

2. LC/TLC responses can surely be obtained.

It is questionable whether an LC-response can be obtained at low voltages as the $\mathrm{LC}$ response at least in some cases must be a special case of the TLC response with the initial measurements carried out above the transition voltage level.

Neither TLC nor LC responses were obtained during the high voltage measurements so for the applied cable samples TLC and LC responses will obviously not be present at lower voltages. Though it may be a theoretical possibility that $\mathrm{LC}$ as well as TLC responses can be obtained at low voltages, the amount of cables which falls into these categories will definitely be much smaller, presumably equal to zero, when measurements are carried out at low voltages. Thus, the only part of the high voltage method which is applicable at low voltages is the ranking of cables with respect to the degree of nonlinearity. Fig. 8 shows the results from various subsequent low voltage sweeps on cable samples P1-P3.

Even though the measurements were carried out at the same voltage, $140 \mathrm{~V}$, the variation was significant compared to the degree of nonlinearity below $8 \mathrm{kV}$ in the high voltage measurements. Thus it must be concluded that the degree of nonlinearity cannot be determined directly from low voltage measurements due to the obtained variation. It is possible that the variation will be less pronounced, if the low voltage measurements are carried out long after the high voltage stress is removed. It may also improve the response to ground the cable or expose it for a while to a lower voltage stress before the measurements are carried out. The latter approach worked with success for the high voltage response. This has not been investigated at low voltages. As it is now, it does not seem likely that the ranking with respect to VDP can be applied directly at low voltages. It is possible that other ways of interpreting a low voltage sweep will be more rewarding. A proposal is given below, though it needs additional verification.

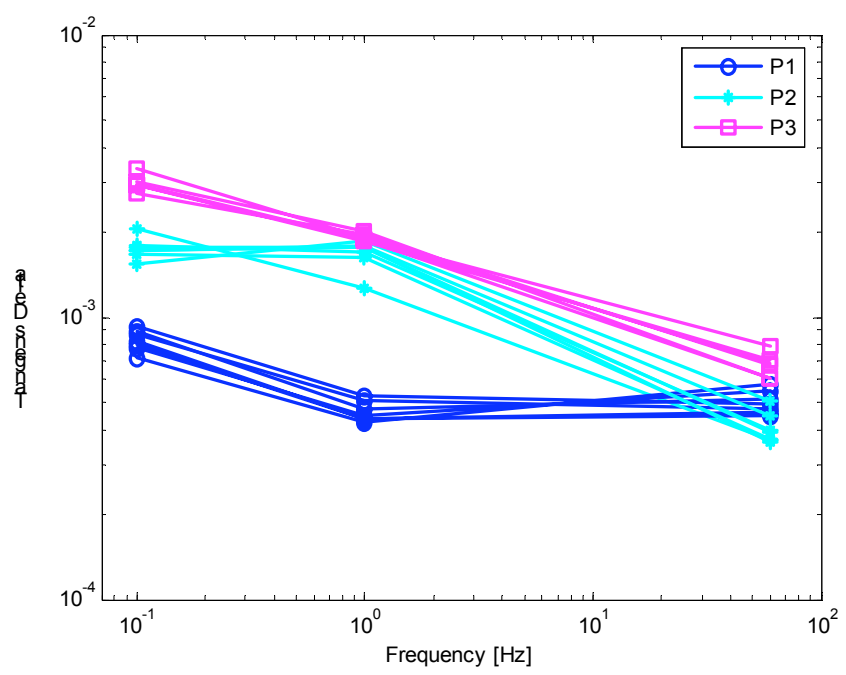

Fig. 8. Several $\tan \delta$-sweeps for P1-P3 using low voltages (140V) at frequencies $60.15 \mathrm{~Hz}, 1 \mathrm{~Hz}$ and $0.1 \mathrm{~Hz}$ for examining the variation in measurements as well as the frequency response.

It has been reported as well as verified through this investigation, that the voltage dependence of water treed XLPE exhibits strong hysteretic effects. More over, the hysteretic effects exhibit time dependence [4]. Thus, independent of the exact explanation for this time dependence, processes are also present in a water treed cable when the high voltage stress is removed. The variation in the measured dissipation factor, even at lower voltages, can therefore be a measure for the amount of processes going on, and thus an indirect measure for the degree of VDP at higher voltages, in case the service voltage has been applied recently. Of course, other factors may also influence on the variation.

When applying the high voltage method, one way to separate the nonlinearity caused by a VDP response from the response obtained by e.g. terminations, is that the degree of VDP is frequency independent. For a possible low voltage method it is also possible that the used measure, for instance the variation in the dissipation factor, is frequency independent unlike the variation caused by other factors. Applying this interpretation method to fig. 8, P1 is estimated as significantly water treed compared to P2 and P3. This is the same conclusion which was drawn from the high voltage measurements - though it cannot be rejected that P3 also would have been estimated as being water treed if the amplifier could have supplied a higher voltage. As the proposed low voltage interpretation method is partly inspired by the obtained results, additional verification is needed.

If future studies do not show that the proposed low voltage method is reliable, it is necessary to do the measurements at high voltage. In some cases this is impractical as one of the main drawbacks of the high voltage method is that the amplifier cannot supply the needed current for long cables even at low frequencies. However, the current drawn by the 
amplifier can theoretically be reduced by connecting an inductor across the amplifier as this will not influence the current and voltage measurements. Ideally the inductor will be variable and of small size. However, the actual size will depend on the cable lengths of interest as the required inductance will decrease with increasing cable length. The modification is illustrated in fig. 9. We regard this kind of compensation as a possible enhancement for future versions of the IDA system with an advanced control scheme.

\section{CONCLUSIONS}

The presented work supports the high voltage method developed at KTH and rejects the hypothesis of measuring the degree of the voltage dependency of the permittivity with the low voltage equipment without additional initiatives. An alternative interpretation of the low frequency response was presented but more experiments are needed to reject or verify this approach. Finally a theoretical improvement of the IDA equipment was proposed.

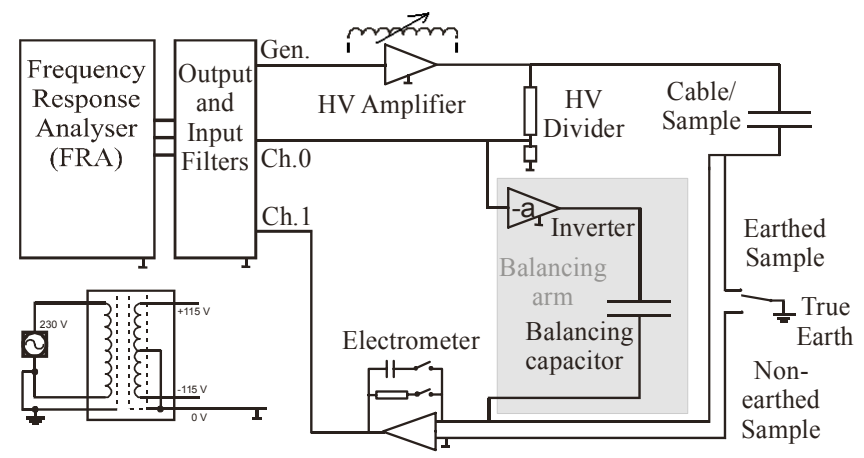

Fig. 9. Drawing showing the principles behind the applied measurement system, though with a theoretical enhancement. Taken from [4] by kind permission and added a variable inductance.

\section{ACKNOWLEDGMENT}

We gratefully acknowledge (in alphabetic order) Jody Levine, John Densley, John Dipaul, John Kuffel, Peter Werelius, Joacim Skoldin, Rick Glowacki and Silvano Rizzetto for their help on several issues.

\section{REFERENCES}

[1] B. Holmgren, Dielectric response, breakdown strength and water tree content of medium voltage XLPE cables, licentiate thesis, $\mathrm{KTH}$, Stockholm, Sweden, ISSN 1100-1593, 1997.

[2] P. Werelius, P. Thärning, R. Eriksson, B. Holmgren, U. Gäfvert, "Dielectric Spectroscopy for Diagnosis of Water Tree Deterioration in XLPE Cables", IEEE Trans. Dielectrics and Electrical Insulation, vol. 8, No. 1, p.27-p.42, February 2001.

[3] S. Hvidsten, Nonlinear Dielectric Response of Water Treed XLPE Cable Insulation, doctoral thesis, NTNU, Trondheim, Norway, ISBN 82-4710433-4, 1999.

[4] P. Werelius, Development and Application of High Voltage Dielectric Spectroscopy for Diagnosis of Medium Voltage XLPE Cables, doctoral thesis, KTH, Stockholm, Sweden, ISSN 1650-674x, 2001. Currently obtainable at: http://www.diva-portal.org/kth/theses/

[5] B. Holmgren, P. Werelius, R. Eriksson, U. Gäfvert, "Dielectric Measurements for Diagnosis of XLPE Cable Insulation“, CIRED 97, Conf. publ. no. 438, IEE 1997.

[6] B. Holmgren, S. Hvidsten, J. Wetterström, U. Grape, "Current Status of Condition Assessment of Water Treed 12 and $24 \mathrm{kV}$ XLPE Cables in Norway and Sweden", Nord-IS 05, Trondheim, Norway, p.71-75.

[7] R. Eriksson, P. Werelius, L. Adeen, P. Johansson, H. Flodqvist, "Condition Based Replacement of Medium Voltage Cables Saves Millions - Case Study Botkyrka”, IEEE Bologna PowerTech Conference, Bologna, Italy, 2003.

[8] Oyegoke, B. and P. Hyvonen, M. Aro, N. Gao, "Application of Dielectric Response Measurement on Power Cable Systems", IEEE Trans. Dielectrics and Electrical Insulation. Vol.10 No. 5, p.862-p.873, February 2003. 